\title{
Thrombotic Thrombocytopenic Purpura
}

National Cancer Institute

\section{Source}

National Cancer Institute. Thrombotic Thrombocytopenic Purpura. NCI Thesaurus. Code C78797.

A coagulation disorder characterized by extensive formation of thrombi in small blood vessels throug hout the body due to low levels of ADAMTS13 protein, and resulting in consumption of circulating platelets, which is characterized by thrombocytopenia, anemia, neurologic changes, and sometimes fever and renal dysfunction. 